\title{
DATOS SOBRE LOS GENEROS ENTONAEMA Y USTULINA (PYRENOMYCETES, XYLARIACEAE)
}

\author{
Felipe Eduardo San Martin \\ Y \\ Pablo Antonio LaVin \\ Biodiversidad Tamaulipeca, A.C. \\ Sierra Hermosa 617 \\ Fracc. Villa Real \\ 87010 Cd. Victoria, Tamaulipas
}

\section{RESUMEN}

Se describen e ilustran los Xylariaceae Entonaema globosum Heim, E. liquescens A. Möll., E. pallidum G. W. Martin., Ustulina cf. brasiliensis Speg., U. deusta (Hoffm.: Fr.) Petrak, U. pavimentosa (Ces.) Cooke, U. sp. SM-739, U. sp. SM-1112 y U. zonata (Lév.) Sacc. Para las especies de cada género recolectadas a la fecha en México, se provee una clave.

\section{ABSTRACT}

The Xylariaceae Entonaema globosum Heim, E. liquescens A. Möll., E. pallidum G. W. Martin, Ustulina cf. brasiliensis Speg., U. deusta (Hoffm.: Fr.) Petrak, U. pavimentosa (Ces.) Cooke, U. sp. SM-739, U. sp. SM-1112, and U. zonata (Lév.) Sacc. are described and illustrated. For the species of each genus collected in Mexico so far, a key is provided.

\section{INTRODUCCION}

Esta contribución compila información original y de la literatura sobre los géneros Entonaema y Ustulina. Dado que el primero es aparentemente raro y su distribución en México es poco conocida y al menos una especie del segundo es fitopatógena, el trabajo pretende dar servicio a los ascomicetólogos y fitopatólogos mexicanos interesados en la taxonomía y biología de estos Xylariaceae destructores de madera.

\section{METODOLOGIA}

La forma de recolectar, herborizar e identificar las especies se puede consultar en San Martín y Rogers (1995) y en San Martín (1996).

Fragmentos de estroma y paredes periteciales de las especies de Entonaema se pusieron en contacto con una gota de solución de $\mathrm{KOH}$ a $10 \%$, colocada en la superficie de un cubreobjetos. El color del o de los pigmentos extraídos se comparó con la tabla de 
colores de Kornerup y Wanscher (1961) y se le asignó la clave correspondiente en ésta, el primer número pertenece a la placa y el número y la letra siguientes a la combinación que da el color en dicha placa.

Los especímenes se encuentran depositados en ITCV, JDR y en el herbario personal de Ascomycetes de Felipe San Martín. Para la designación de herbarios se sigue a Holmgren et al. (1990).

\section{EL GENERO ENTONAEMA A. MÖLL.}

Alfred Möller (1901) erigió Entonaema para ubicar las especies E. mesenterica A. Möll. y E. liquescens A. Möll. Entonaema se caracteriza por sus estromas pulvinados, hemisféricos o globosos, lobados a irregulares, con una o varias cavidades ocupadas por una substancia gelatinosa acuosa en la madurez que desaparece en la senectud para dar paso a oquedades. El endostroma es gelatinoso acuoso cuando fresco, correoso y duro cuando seco. Los peritecios son carbonosos, monósticos, dispuestos bajo una capa externa que varía, según la especie, de color gris a anaranjado. Los ascos son cilíndricos, estipitados, con un anillo apical amiloide, discoide a rectangular. Las ascosporas unicelulares son de color café, elipsoides inequilaterales a más o menos rectangulares, con línea germinal recta, corta o a todo lo largo de la parte coloreada de la ascospora.

El anamorfo de Entonaema es asignable a Nodulisporium Preuss. Tal estado conidial lo comparte con Hypoxylon y Daldinia, por lo que se considera a los tres géneros estrechamente relacionados pero claramente distintos (Rogers, 1982; Ju y Rogers, 1996; Ju et al., 1997).

Al presente las especies aceptadas de Entonaema son seis (Rogers, 1981; Rogers et al., 1996) a saber: E. cinnabarinum (Cooke et Massee) Lloyd, E. dengii J. D. Rogers, E. globosum Heim, E. liquescens Möll., E. moluccanum J. D. Rogers y E. pallidum G. W. Martin.

\section{CLAVE PARA LAS ESPECIES DE ENTONAEMA RECOLECTADAS EN MEXICO}

1 Superficie de color blanco a grisáceo. Ostiolos finamente papilados. Pigmento estromático extraído con $\mathrm{KOH}$ de color grisáceo amarillento a oliváceo (3-3B). Ascosporas de color café a café obscuro, de (10.5)11-12(14.5) x 5.5-6(6.5) $\mu \mathrm{m}$

E. pallidum

1 Superficie estromática de color amarillo o anaranjado. Con otra combinación de caracteres.

2 Estroma de 1.2-4 cm de largo x 0.9-2.7 cm de ancho x 0.7-1.5 cm de alto. Superficie de color amarillo vívido a anaranjado pálido (2-8A), con una capa de color rojo sangre abajo. Ostiolos umbilicados. Pigmento estromático extraído con $\mathrm{KOH}$ de color anaranjado rojizo a rojo sangre (8-8B). Ascosporas de color café claro a café,

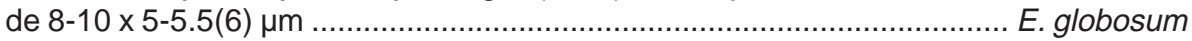

2 Estroma de $3-21 \mathrm{~cm}$ de largo $\times 2-17.5 \mathrm{~cm}$ de ancho $\times 1.5-5.5 \mathrm{~cm}$ de alto. Superficie de color amarillo a amarillo pálido (8-5A). Ostiolos umbilicados a ligeramente papilados. Pigmento estromático extraído con $\mathrm{KOH}$ de color rojo amarillento (6-7A). Ascosporas de color café, de 10-12 x 5.5-7 $\mu \mathrm{m}$

E. liquescens 
Entonaema globosum Heim, Bull. Soc. Myc. France 76: 121. 1960 (como globosa). Fig. 1 A.

Estroma globoso a irregular, sésil, de $1.2-4 \mathrm{~cm}$ de largo $x$ 0.9-2.7 $\mathrm{cm}$ de ancho $\mathrm{x}$ $0.7-1.5 \mathrm{~cm}$ de alto, externamente de color amarillo vívido a anaranjado pálido (2-8A) con una capa de color rojo sangre (8-8B) inmediatamente abajo, el interior al principio lleno de un líquido gelatinoso acuoso, después hueco con las paredes internas córneas, con pigmentos extraídos al contacto con $\mathrm{KOH}$ de color anaranjado rojizo a rojo sangre (8-8B). Textura carbonosa a dura. Superficie lisa o muy arrugada, pruinosa, con hendiduras discretas. Peritecios globosos, de 0.1-0.3 mm de diámetro. Ostiolos umbilicados. Ascos cilíndricos, con ocho ascosporas dispuestas irregularmente y estípites muy largos, de 136-191 $\mu \mathrm{m}$ de longitud total x 8-9 $\mu \mathrm{m}$ de ancho, la parte esporígena de 48-59 $\mu \mathrm{m}$, con anillo apical amiloide rectangular a discoide, de $1 \mu \mathrm{m}$ de alto $\times 2.5 \mu \mathrm{m}$ de ancho. Ascosporas de color café claro a café, elipsoidales con extremos redondeados, unigutuladas a bigutuladas, de 8-10 x 5-5.5(6) $\mu \mathrm{m}$, con línea germinal recta a todo lo largo del propágulo.

Espécimen estudiado: Campeche, municipio de Escárcega, Estación Experimental Forestal Eduardo Sangri Serrano, sobre madera de Nectandra salicifolia (H. B. K.) Nees, (laurelillo) en un bosque tropical alto subperennifolio, 9.XI.1988, San Martín 1171 (ITCV: neotipo, JDR: duplicado del neotipo).

Notas: Entonaema globosa fue propuesta por Heim (1960) con base en material recolectado en la región mazateca de Oaxaca. Posteriormente, en el Index of Fungi \# 3 de 1961 el epíteto específico se corrigió ortográficamente por globosum. Dado que el material tipo depositado en $\mathrm{P}$ carece de ascos y ascosporas, Rogers et al. (1996) neotipificaron la especie.

De acuerdo con Rogers (1981), E. globosum es una especie cercana a $E$. cinnabarinum, pero esta última presenta una capa de gránulos de color anaranjado bajo el ectostroma y el tamaño de sus ascosporas es de (9)10-13 x 5-7 $\mu \mathrm{m}$.

Entonaema liquescens A. Möll., Phycomyc. Ascomyc. Bras., 306. 1901. Fig. 1B.

Estroma globoso a irregular, sésil, de $3-21 \mathrm{~cm}$ de largo $\times 2-17.5 \mathrm{~cm}$ de ancho $\mathrm{x}$ 1.5-5.5 cm de alto, externamente de color amarillo a amarillo pálido (8-5A), café obscuro (5-5F) inmediatamente abajo, el interior al principio lleno de una substancia acuosa gelatinosa, después hueco, de consistencia correosa, pigmento estromático extraído con $\mathrm{KOH}$ de color rojo amarillento (6-7A). Textura correosa. Superficie lisa a arrugada, pruinosa. Peritecios globosos, de 0.4-0.7 mm de diámetro. Ostiolos umbilicados a ligeramente papilados. Ascos cilíndricos, octosporados en una serie oblicua o biseriada, con estípites muy largos, de 112-128 $\mu \mathrm{m}$ de longitud total x 6.5-7.5 $\mu \mathrm{m}$ de ancho, la parte esporígena de 44-60 $\mu \mathrm{m}$, con anillo apical amiloide discoide a cúbico, de $0.8 \mu \mathrm{m}$ de alto $\times$ 3-4 $\mu \mathrm{m}$ de ancho. Ascosporas de color café, elipsoides a ligeramente inequilaterales, bigutuladas con extremos redondeados, de 10-12 x 5.5-7 $\mu \mathrm{m}$, con línea germinal inconspicua, recta, algo menor que la longitud total del propágulo. 
$\therefore, 08.80$.<smiles></smiles> 
Especímenes estudiados: Jalisco, municipio de Tamazula, San Francisco, sobre madera en un bosque de olivos (sic), 15.VIII.1985, Arreaga Pérez 46 (IBUG). Tamaulipas, municipio de Ocampo, colonia Santa María Guadalupe, sobre madera de una dicotiledónea en un bosque tropical mediano subcaducifolio, 16.V.1988, San Martín 634 (ITCV, JDR).

Notas: El material recolectado en México concuerda con la descripción que para $E$. liquescens proveyó Rogers (1981). La especie ha sido registrada de toda América (Rogers, 1981), y Heredia (1989) fue la primera en comunicar sobre su existencia en México.

Entonaema pallidum G. W. Martin, Mycologia 30: 431. 1938 (como pallida). Fig. 1C.

Estroma globoso a irregular, pulvinado, de $1.2-4 \mathrm{~cm}$ de largo $\times 0.9-2.7 \mathrm{~cm}$ de ancho $\times$ 0.7-1.5 cm de alto, externamente de color blanquecino a gris (3-1B), interior café obscuro (5-5F), al principio llenos de una substancia acuosa gelatinosa, después huecos. Textura correosa. Superficie irregular debido a arrugas y protrusiones periteciales prominentes. Pigmento estromático extraído con $\mathrm{KOH}$ de color grisáceo amarillento a oliváceo (3-3B). Peritecios globosos, de 0.4-0.7 mm de diámetro. Ostiolos finamente papilados. Ascos cilíndricos, no intactos, con anillo apical amiloide discoide a cúbico, de 2-2.5 $\mu \mathrm{m}$ de ancho x 1-1.5 $\mu \mathrm{m}$ de alto. Ascosporas de color café a café obscuro, elipsoides a elipsoides inequilaterales con extremos redondeados a estrechamente redondeados, de (10.5)11-12(14.5) x 5.5-6(6.5) $\mu \mathrm{m}$, con línea germinal recta, evidente, a todo lo largo del propágulo.

Especímenes estudiados: Chiapas, municipio de Ocozocuautla, reserva ecológica Laguna Bélgica, sobre madera en un bosque tropical bajo subcaducifolio, VII.1985, San Martín 410 (ITCV, JDR). Tamaulipas, cabecera municipal de Gómez Farías, sobre madera en un bosque tropical mediano subcaducifolio, 16.VII.1987, Martínez 4 y Baldazo 9 (ITCV, JDR).

Notas: Los caracteres del material mexicano concuerdan con los que conforman la descripción que, para la aparentemente rara Entonaema pallidum, proveyó Martin (1938) con material recolectado en Panamá. La especie ha sido citada también de Trinidad y Tobago (Rogers, 1981); Medel y Chacón (1992) la registraron por vez primera para la micoflora mexicana del estado de Chiapas.

\section{EL GENERO USTULINA TUL.}

Los autores aceptan al género Ustulina en el sentido de Tulasne (In Saccardo, 1882) quien lo caracterizó como un esferial con estromas superficiales, repando pulvinados, muy gruesos, carbonosos, negros y con el interior hueco cuando maduros. Miller (1961) ubicó a Ustulina como sinónimo de Hypoxylon debido, en su opinión, a la ausencia de diferencias entre ambos géneros. Para Laessøe (1994) Ustulina es sinónimo de Kretzschmaria en virtud de la afirmación de Ko et al. (1982), quienes aseguraron que factores del medio determinan 
formas estromáticas transicionales entre Hypoxylon y Kretzschmaria. Jong y Rogers (1972) consideraron en sus notas sobre Hypoxylon deustum (Hoff. ex Fr.) Grev. que Ustulina puede ser un género legítimo, debido a que el arreglo de tipo esporodoquio de su anamorfo se asemeja más a Xylaria que a Hypoxylon. Lo anterior los llevó a definir a Ustulina como intermedio entre Xylaria e Hypoxylon, por lo que para ellos el binomio que indica claramente la posición taxonómica del hongo es la especie tipo Ustulina deusta (Hoffm. ex. Fr.) Petrak.

De acuerdo con Petrini y Petrini (1985), el anamorfo de H. deustum es asignable a Hadrotrichum Fuckel. Para Ju y Rogers (1996) el anamorfo de Ustulina es del tipo Geniculosporium Chesters et Greenhalgh.

De las especies de Ustulina presentes en México, destaca U. zonata (Lév) Sacc. por su condición de fitopatógeno (Wilkins, 1933, 1936; como U. vulgaris Tul.). A este hongo se le ha detectado como causante de pérdidas importantes en huertas citrícolas de Veracruz, Tamaulipas y Nuevo León.

\section{CLAVE PARA LAS ESPECIES DE USTULINA RECOLECTADAS EN MEXICO}

1 Estromas en madera de monocotiledóneas, orbiculares, de 1.7-2 mm de alto x 3.5-12 $\mathrm{mm}$ de ancho. Ascosporas elipsoides inequilaterales a naviculares con extremos estrechos a muy agudos y algo curvos, de (40)41-44(45) x 8.5-9(10) $\mu \mathrm{m}$

Ustulina cf. brasiliensis

1 Estromas en madera de dicotiledóneas 2

2 Estromas orbiculares, de $0.5-1.5 \mathrm{~cm}$ de largo $\times 0.5-1 \mathrm{~cm}$ de ancho $\times 0.3-0.5 \mathrm{~cm}$ de alto. Peritecios de 0.8-1 mm de alto x 0.6-0.8 mm de ancho. Ascosporas elipsoides inequilaterales, con extremos estrechamente redondeados a algo agudos, de (33.5)36-41(43) x 7-11(12) $\mu \mathrm{m}$, con línea germinal recta, corta, alcanzando menos de $3 / 4$ la longitud total del propágulo ............... Ustulina deusta

2 Estromas y ascosporas con otra combinación de caracteres .................................. 3

3 Ascosporas menores de $40 \mu \mathrm{m}$ de largo ......................................................... 4

3 Ascosporas mayores de $40 \mu \mathrm{m}$ de largo ..................................................... 5

4 Estromas repando pulvinados, de $3-7.5 \mathrm{~cm}$ de largo $\times 2-5 \mathrm{~cm}$ de ancho $x$ 0.1-0.2 cm de alto. Ascosporas elipsoides inequilaterales a crescénticas, con extremos estrechos, frecuentemente constreñidos, de color casi negro, de 25-29(30) x 8-12 $\mu \mathrm{m}$, con línea germinal recta, casi a todo lo largo del propágulo Ustulina zonata

4 Estromas orbiculares, de 1.5-3 cm de largo x 1.5-2 cm de ancho x 0.1-0.2 cm de alto. Ascosporas elipsoides inequilaterales con extremos redondeados a estrechamente redondeados, de color café a café obscuro, de (29)30-38(39) x 7-9(9.5) $\mu \mathrm{m}$, con línea germinal recta, casi a todo lo largo del propágulo Ustulina sp. SM-1112

5 Estromas pulvinados, de $3-5.5 \mathrm{~cm}$ de largo $\times 1.5-3.5 \mathrm{~cm}$ de ancho $\times$ 0.7-0.9 $\mathrm{cm}$ de alto. Ascosporas elipsoides inequilaterales a naviculares con extremos redondeados a estrechamente redondeados, de color café obscuro a casi negro, de (42.5)43-48(49) x 9-11(12) $\mu \mathrm{m}$, con línea germinal recta, casi a todo lo largo del propágulo Ustulina pavimentosa 
5 Estromas orbiculares, de 0.8-1.5 cm de diámetro $\times$ 0.1-0.2 cm de alto. Ascosporas elipsoides a elipsoides inequilaterales con extremos redondeados, de color casi negro, de (53.5)54-60(65) x (17)18-24(25.5) $\mu \mathrm{m}$, con línea germinal recta, a todo lo largo del propágulo

Ustulina sp. SM-739

Ustulina cf. brasiliensis Speg., Anales Soc. Ci. Argent. 12: 50. 1881. Fig. 1D.

Estromas orbiculares, sésiles o con un rudimento de estípite en el centro, de 4.5-12 mm de largo x 3.5-12 mm de ancho x 1.7-2 mm de alto; al principio con la parte externa de color café, después negruzca, internamente negros, huecos; superficie lisa con hendiduras y papilas periteciales. Textura dura. Peritecios subglobosos a piriformes, de 1-1.5 mm de alto x $1.5 \mathrm{~mm}$ de ancho, ostiolos cónico papilados, muy espaciados. Ascos no observados. Ascosporas de color café a café obscuro, elipsoides inequilaterales a naviculares, con extremos estrechos a muy agudos y algo curvos, de (40)41-44(45) x 8.5-9(10) $\mu \mathrm{m}$, con línea germinal recta, corta, alcanzando menos de 3/4 la longitud total del propágulo, en el lado cóncavo.

Espécimen estudiado: Chiapas, ejido Boca de Chajul, municipio de Ocosingo, sobre madera de la monocotiledónea llamada chocho en un acahual, 28.V.1988, San Martín 824 (ITCV).

Notas: El espécimen estudiado está en la senectud y los intentos por cultivarlo fueron infructuosos. La ubicación de este hongo bajo el nombre de $U$. brasiliensis es provisional, debido a que no se encontró en la literatura otra especie cuyos caracteres concordaran mejor con los de él. Asimismo, el taxon fue recolectado sobre madera de una monocotiledónea y en la descripción que para $U$. brasiliensis dio Spegazzini (In Saccardo, 1882) se mencionan como hospedantes a troncos podridos sin precisar la clase.

Ustulina deusta (Hoffm.: Fr.) Petrak, Ann. Mycol. 19: 279. 1921. Fig. 1E.

Estromas orbiculares, sésiles, adheridos al substrato por un conectivo central, de $0.5-1.5 \mathrm{~cm}$ de largo x 0.5-1 cm de ancho x 0.3-0.5 cm de alto, de crecimiento restringido; al principio con el exterior de color blanquecino, después café a café obscuro, internamente negruzco con una capa fibrosa de color café abajo de la capa de peritecios, huecos con la edad; superficie lisa o con hendiduras y papilas periteciales, a veces zonada. Textura al principio correosa, después carbonosa. Peritecios ovales a rectangulares debido a mutua presión, monósticos, de 0.8-1 $\mathrm{mm}$ de alto x 0.6-0.8 $\mathrm{mm}$ de ancho. Ostiolos finamente papilados, cónicos. Ascos cilíndricos, octosporados en una serie lineal, con estípites cortos, de 240-320 $\mu \mathrm{m}$ de longitud total x 10-15 $\mu \mathrm{m}$ de ancho, la parte esporígena de 190-260 $\mu \mathrm{m}$, con un anillo apical amiloide, rectangular, de 8-10 $\mu \mathrm{m}$ de alto x 4-6 $\mu \mathrm{m}$ de ancho. Ascosporas de color café a café obscuro, elipsoides inequilaterales con extremos estrechamente redondeados a algo agudos, de (33.5)36-41(43) x 7-11(12) $\mu \mathrm{m}$, con línea 
germinal recta, corta, alcanzando menos de 3/4 la longitud total del propágulo, por el lado cóncavo.

Especímenes estudiados: Chiapas, municipio de Jaltenango, camino El TriunfoSanta Rita, sobre madera de un elemento ripario de un bosque mesófilo de montaña, 7.VI.1988, San Martín 667 (ITCV, JDR), municipio de Mapastepec, camino El Triunfo-Paval, sobre madera de una dicotiledónea en una selva tropical mezclada con elementos de bosque mesófilo de montaña, 9.VI.1988, San Martín 739B (ITCV, JDR). Tamaulipas, municipio de Gómez Farías, ejido Julilo, sobre madera de encino popotillo en un bosque mesófilo de montaña, alt. 1465 m, 18.VIII.1995, San Martín $5019 T$ (ITCV).

Notas: El concepto que los autores tienen de $U$. deusta se circunscribe a formas estromáticas presentes en México en bosque mesófilos de montaña, de crecimiento restringido, con ascosporas de extremos estrechamente redondeados a algo aguzados y línea germinal recta, corta y evidente.

Ustulina pavimentosa (Ces.) Cooke, Grevillea 11: 126. 1883. Fig. 1F.

Estromas pulvinados, superficiales, de $3-5.5 \mathrm{~cm}$ de largo $\times 1.5-3.5 \mathrm{~cm}$ de ancho $\mathrm{x}$ 7-9 mm de alto, externamente de color café a café obscuro, internamente negro, huecos con la edad. Textura dura pero frágil. Superficie no lisa con hendiduras y papilas periteciales. Peritecios ovales a oblongo comprimidos, monósticos, de 3-4 mm de alto x 1-1.5 mm de ancho. Ostiolos prominentes, cónico papilados. Ascos no observados. Ascosporas de color café obscuro a casi negro, elipsoides inequilaterales a naviculares con extremos redondeados a estrechamente redondeados, de (42.5)43-48(49) x 9-11(12) $\mu \mathrm{m}$, con línea germinal recta, casi a todo lo largo del propágulo.

Espécimen examinado: Chiapas, municipio de Ocosingo, reserva de la biosfera Montes Azules, sobre madera de una dicotiledónea en un bosque tropical alto perennifolio, 29.V.1988, San Martín 838 (ITCV, JDR).

Notas: La colección mexicana difiere del material tipo de U. pavimentosa, procedente de Borneo (ahora Kalimantán) -descrita el siglo pasado por Cesati como Hypoxylon pavimentosum Ces. y completada su descripción por Miller (1961)- en que tiene estromas más grandes y gruesos, en que sus peritecios son más grandes y no están en contacto con el substrato. El resto de sus caracteres son como los de U. pavimentosa. Martin (1976) consideró a esta especie como Kretzschmaria pavimentosa (Ces.) P. Martin. Ustulina pavimentosa se ha citado de Belice, Kalimantán y Sudáfrica.

Ustulina sp. SM-739. Fig. $1 \mathrm{G}$.

Estromas orbiculares, sésiles, adheridos al substrato por un conectivo central, de crecimiento restringido, de 0.8-1.5 cm de diámetro $x$ 0.1-0.2 cm de alto; 
externamente de color café a café obscuro, internamente café obscuro, con una capa fibrosa de color café bajo la línea de peritecios; superficie lisa o con hendiduras y papilas periteciales. Textura dura. Peritecios ovales, monósticos, de 0.9-1 mm de diámetro. Ostiolos discretos a hemisféricamente papilados. Ascos no vistos. Ascosporas de color casi negro, elipsoides a elipsoides inequilaterales, con extremos redondeados, de (53.5)54-60(65) x (17)18-24(25.5) $\mu \mathrm{m}$, con línea germinal recta, casi a todo lo largo del propágulo, por el lado cóncavo.

Espécimen estudiado: Chiapas, municipio de Mapastepec, camino El Triunfo-Paval, sobre madera en un bosque mesófilo de montaña, 9.VI.1988, San Martín 739 (ITCV).

Nota: Al parecer este hongo representa una especie no descrita. No es conveniente proponerla como nueva porque el material carece de ascos y se desconoce su estado anamórfico.

Ustulina sp. SM-1112. Fig. $1 \mathrm{H}$.

Estromas orbiculares, adheridos al substrato con toda la base o mediante un conectivo central, de crecimiento restringido, de $1.5-3 \mathrm{~cm}$ de largo $\times 1.5-2 \mathrm{~cm}$ de ancho $x$ 0.1-0.2 cm de alto, externamente de color café claro, internamente negro. Textura dura. Superficie lisa o con hendiduras y ostiolos. Peritecios ovales a casi rectangulares por presión mutua. Ostiolos prominentemente papilados, cónicos o hemisféricos. Ascos cilíndricos, octosporados en una serie lineal o parcialmente biseriados, con estípites cortos, de 277.5-317 $\mu \mathrm{m}$ de longitud total $\times$ 9.5-10 $\mu \mathrm{m}$ de ancho, la parte esporígena de 217.5-256 $\mu \mathrm{m}$, con un anillo apical amiloide rectangular, de 5.5-7 $\mu \mathrm{m}$ de alto $\times$ 4-5 $\mu \mathrm{m}$ de ancho. Ascosporas elipsoides inequilaterales con extremos redondeados a estrechamente redondeados, de color café a café obscuro, de (29)30-38(39) x 7-9(9.5) $\mu \mathrm{m}$, con línea germinal recta, casi a todo lo largo del propágulo, por el lado cóncavo.

Especímenes examinados: Chiapas, municipio de Ocosingo, ejido Boca de Chajul, sobre madera de una dicotiledónea en un acahual, 27.V.1988, San Martín 773C (ITCV, JDR). Oaxaca, Temazcal, en madera de un bosque tropical mediano subperennifolio, 8.X.1988, San Martín 1112 (ITCV, JDR). Tamaulipas, municipio de Jaumave, El Malacate, sobre madera en un bosque mesófilo de montaña, 31.III.1988, Baldazo 207 (ITCV, JDR); municipio de Gómez Farías, rancho El Cielo, sobre madera en un bosque mesófilo de montaña, 22.VII.1988, Chacón 371 (ITCV, JDR); alrededores de Gómez Farías, sobre madera en una selva mediana subcaducifolia, 2.IX.1988, San Martín $1047 T$ (ITCV, JDR); municipio de Ocampo, colonia Santa María Guadalupe, sobre madera en un bosque tropical mediano subcaducifolio, San Martín 639 (ITCV, JDR).

Notas: Esta especie es desconocida para los autores. Según Rogers (comunicación personal), las recolectas San Martín 639, 773C y 1112 y Chacón 371 corresponden a Hypoxylon sandvicense Reichardt. De acuerdo con Ju y Rogers (1996) H. sandvicense es una especie de Ustulina. 
Las recolectas Baldazo 207 y San Martín $1047 T$ tienen estromas más efusos y ascosporas menores a las que presentan San Martín 639, 773C y 1112 y Chacón 371. Quizás se trate de una variante de las formas comprendidas en U. sp. SM-1112.\}

Ustulina zonata (Lév.) Sacc., Syll. Fung. I: 352. 1882. Fig. 11.

Estromas repando pulvinados, superficiales, de crecimiento irrestricto, de $3-7.5 \mathrm{~cm}$ de largo x 2-5 cm de ancho x 0.1-0.2 cm de alto, externamente de color café obscuro a negro, internamente negruzco con una capa blanquecina abajo de los peritecios. Textura dura. Superficie lisa con ostiolos y protrusiones periteciales. Peritecios ovales, monósticos, de 1.2-1.5 mm de alto x 0.9-1 mm de ancho. Ostiolos inconspicuos a hemisféricamente papilados. Ascos no observados. Ascosporas de color casi negro, elipsoides inequilaterales a crescénticas con extremos estrechos, frecuentemente constreñidos, de 25-29(30) x 8-12 $\mu \mathrm{m}$, con línea germinal recta, casi a todo lo largo del propágulo.

Especímenes examinados: Chiapas, cabecera municipal de Tuxtla Gutiérrez, Zoológico Miguel Alvarez Del Toro, sobre madera en un bosque tropical alterado, 22.V.1988, San Martín 712 (ITCV, JDR). Quintana Roo, municipio de José María Morelos, rancho Zafarrancho, sobre madera de un arbol vivo de Citrus sp. en una huerta, 6.II.1986, Guevara 733 (ITCV, JDR). Veracruz, municipio de Catemaco, estación biológica Los Tuxtlas de la Universidad Nacional Autónoma de México, sobre madera de una dicotiledónea en un acahual, 4.XI.1988, San Martín 1380V (ITCV, JDR), municipio de Papantla, sobre madera de Citrus sinensis (L.) Osbeck, causando una enfermedad tipo muerte descendente, 26.II.1987, Gómez 1 (ITCV, JDR).

Notas: Ustulina zonata ha sido considerada como una forma tropical de U. deusta (Wilkins, 1933). Los autores concuerdan con Wakefield (In Miller, 1961) en cuanto a considerar a $U$. zonata distinta de $U$. deusta, sobre la base del mayor grosor de sus ascosporas y su patogenicidad.

\section{AGRADECIMIENTOS}

Los autores patentizan su agradecimiento a dos revisores anónimos que mejoraron substancialmente el manuscrito. Asimismo, se reconoce la participación del Dr. Jack. D. Rogers de la Universidad Estatal de Washington, por contribuir con sus buenos oficios a lograr financiamiento para el trabajo de campo por parte de la Fundación Nacional de Ciencias de los Estados Unidos de América, por revisar el escrito y llamar nuestra atención sobre la presencia en México de Ustulina zonata y posiblemente de Hypoxylon (= Ustulina) sandvicense. 


\section{LITERATURA CITADA}

Heim, R. 1960. Quelques ascomycetes remarquables, III. Le genre Entonaema Möell. du Mexique. Bull. Soc. Myc. France 76: 121-129.

Heredia, G. 1989. Estudio de los hongos de la reserva de la biosfera El Cielo, Tamaulipas. Consideraciones sobre la distribución y ecología de algunas especies. Acta Bot. Mex. 7: 1-17.

Holmgren, P. K., N. H. Holmgren y L. C. Barnett. 1990. Index Herbariorum. I. The herbaria of the world. 8th ed. New York Botanical Garden. Bronx, Nueva York. 693 pp.

Jong, S. C. y J. D. Rogers. 1972. Illustrations and descriptions of conidial states of some Hypoxylon species. Wash. State Agric. Exp. Sta. Bull. 71. 51 pp.

Ju, Y.-M. y J. D. Rogers. 1996. A revision of the genus Hypoxylon. The Mycological Society of America. Mycologia Memoir No. 20 and the American Phytopathological Society of America. St. Paul, Minnesota. $365 \mathrm{pp}$.

Ju, Y.-M., J. D. Rogers y F. San Martín. 1997. A revision of the genus Daldinia. Mycotaxon 61: 243293.

Ko, W. H., W. C. Ho y R. K. Kunimoto. 1982. Relation of Kretzschmaria clavus to hypoxyloid stromata on diseased macadamia tissues. Phytopathology 72: 1357-1358.

Kornerup, A. y J. A. Wanscher. 1961. Methuen handbook of colour. Methuen and Co. Londres. 243 pp.

Laessøe, T. 1994. Index ascomycetum 1. Xylariaceae. Systema Ascomycetum 13: 43-111.

Martin, G. W. 1938. New or noteworthy fungi from Panama and Colombia. Mycologia 30: 431-441.

Martin, P. 1976. Studies in the Xylariaceae: supplementary note. J. S. African Bot. 42: 71-83.

Medel, R. y S. Chacón. 1992. Ascomycetes lignícolas de México III. Algunos Sphaeriales. Int. J. Mycol. Lichenol. 5: 253-260.

Miller, J. H. 1961. A monograph of the world species of Hypoxylon. Univ. Georgia Press. Athens. 158 pp.

Möller, A. 1901. Phycomyceten und Ascomyceten Untersuchungen aus Brasilien. G. Fischer, Jena. $310 \mathrm{pp}$.

Petrini, L. E. y O. Petrini. 1985. Xylariaceous fungi as endophytes. Sydowia 38: 216-234.

Rogers, J. D. 1981. Sarcoxylon and Entonaema (Xylariaceae). Mycologia 73: 28-61.

Rogers, J. D. 1982. Entonaema liquescens: description of the anamorph and thoughts on its systematic position. Mycotaxon 15: 500-506.

Rogers, J. D., F. San Martín y Y.-M. Ju. 1996. Mexican Fungi: Xylaria entosulphurea sp. nov. and neotypification of Entonaema globosum. Mycotaxon 58: 483-487.

Saccardo, P. A. 1882. Sylloge fungorum omnium hucusque cognitorum. I. Patavii. 768 pp.

San Martín, F. 1996. Una nueva variedad de Mycopepon smithii (Ascomycetes, Pleosporales). Acta Bot. Mex. 35: 9-12.

San Martín, F. y J. D. Rogers. 1995. Notas sobre la historia, relaciones de hospedante y distribución del género Xylaria (Pyrenomycetes, Sphaeriales) en México. Acta Bot. Mex. 30: 21-40.

Wilkins, W. H. 1933. Studies in the genus Ustulina- with special reference to parasitism I. Introduction, survey of previous literature and host index. Trans. Br. Mycol. Soc. 18: 320-345.

Wilkins, W. H. 1936. Studies in the genus Ustulina- with special reference to parasitism. Trans. Br. Mycol. Soc. 20: 133-157. 